\title{
Performance evaluation of a very high resolution small animal PET imager using silicon scatter detectors
}

\author{
Sang-June Park ${ }^{1}$, W Leslie Rogers ${ }^{1}$, Sam Huh ${ }^{1}$, Harris Kagan ${ }^{2}$, \\ Klaus Honscheid ${ }^{2}$, Don Burdette ${ }^{2}$, Enrico Chesi ${ }^{3}$, Carlos Lacasta ${ }^{4}$, \\ Gabriela Llosa ${ }^{4}$, Marko Mikuz ${ }^{5}$, Andrej Studen ${ }^{5}$, Peter Weilhammer ${ }^{3,6}$ \\ and Neal H Clinthorne ${ }^{1}$ \\ ${ }^{1}$ Division of Nuclear Medicine, University of Michigan, Ann Arbor, MI, USA \\ 2 Department of Physics, Ohio State University, Columbus, OH, USA \\ ${ }^{3}$ CERN, Geneva, Switzerland \\ ${ }^{4}$ IFIC/University of Valencia, Valencia, Spain \\ ${ }^{5}$ Department of Experimental Particle Physics, Institute Jozef Stefan/University of Ljubljana, \\ Ljubljana, Slovenia \\ ${ }^{6}$ Department of Physics, University of Perugia, Perugia, Italy \\ E-mail: sangjune@umich.edu
}

Received 19 October 2006, in final form 22 January 2007

Published 30 April 2007

Online at stacks.iop.org/PMB/52/2807

\begin{abstract}
A very high resolution positron emission tomography (PET) scanner for small animal imaging based on the idea of inserting a ring of high-granularity solidstate detectors into a conventional PET scanner is under investigation. A particularly interesting configuration of this concept, which takes the form of a degenerate Compton camera, is shown capable of providing sub-millimeter resolution with good sensitivity. We present a Compton PET system and estimate its performance using a proof-of-concept prototype. A prototype single-slice imaging instrument was constructed with two silicon detectors $1 \mathrm{~mm}$ thick, each having $5121.4 \mathrm{~mm} \times 1.4 \mathrm{~mm}$ pads arranged in a $32 \times$ 16 array. The silicon detectors were located edgewise on opposite sides and flanked by two non-position sensitive BGO detectors. The scanner performance was measured for its sensitivity, energy, timing, spatial resolution and resolution uniformity. Using the experimental scanner, energy resolution for the silicon detectors is $1 \%$. However, system energy resolution is dominated by the $23 \%$ FWHM BGO resolution. Timing resolution for silicon is $82.1 \mathrm{~ns}$ FWHM due to time-walk in trigger devices. Using the scattered photons, time resolution between the BGO detectors is 19.4 ns FWHM. Image resolution of $980 \mu \mathrm{m}$ FWHM at the center of the field-of-view (FOV) is obtained from a $1 \mathrm{D}$ profile of a $0.254 \mathrm{~mm}$ diameter ${ }^{18} \mathrm{~F}$ line source image reconstructed using the conventional 2D filtered back-projection (FBP). The $0.4 \mathrm{~mm}$ gap between two line sources is resolved in the image reconstructed with both FBP and the maximum likelihood expectation maximization (ML-EM)
\end{abstract}


algorithm. The experimental instrument demonstrates sub-millimeter resolution. A prototype having sensitivity high enough for initial small animal images can be used for in vivo studies of small animal models of metabolism, molecular mechanism and the development of new radiotracers.

(Some figures in this article are in colour only in the electronic version)

\section{Introduction}

The demand for a very high resolution positron emission tomography (PET) instrument is motivated by the need to perform non-invasive studies of small animal models of metabolism, disease mechanisms and physiological processes for potential human application. Mice are often used as experimental animals to determine a number of important variables such as organ or tumor-specific uptake, maximum allowable pharmaceutical dose, and the kinetics of tracer uptake and washout for various organs and tumors. Genetically modified mice using knock-outs, knock-ins and transgenic technologies are especially useful for in vivo testing of drug discovery and development (Nichol and Kim 2001, Thanos et al 2002, Britz-Cunningham et al 2003).

Despite the suitability of mice for biomedical research, it remains challenging to investigate very small size organs such as thyroid (1-7 $\mu \mathrm{l})$ and adrenal $(3-40 \mu \mathrm{l})$ and small functional regions in brain (400-800 $\mu \mathrm{l})$ and kidney $(100-500 \mu \mathrm{l})$ with a 1-2 mm resolution typical of the current generation of animal PET imagers (Cherry 1997, Del Guerra 1998, Chatziioannou 1999, 2001, Correia et al 1999, Huber and Moses 1999, Weber et al 2000, Tai et al 2001, 2003b, 2005, Seidel et al 2003). Existing instruments based on scintillation detectors have difficulty achieving the resolution limit imposed by positron range and annihilation photon acollinearity because of inherent resolution and sensitivity losses resulting from Compton scatter, coherent scatter within the detector, and the penetrating nature of annihilation photons. Moreover, it can be difficult to construct scintillation detectors using large numbers of discrete small crystals.

As a new approach to achieve sub-millimeter image resolution with good counting sensitivity, the concept of using a high spatial resolution solid-state detector as an insert into a more conventional PET ring of scintillation detectors has been proposed for small animal PET (Clinthorne et al 2000, Park et al 2001a, 2001b, 2002, 2004a, 2004b, 2005). Recently, similar concepts have been investigated by other researchers (Tai et al 2003a, Janecek et al 2004). One of the more intriguing configurations of the instrument is a 'degenerate' Compton camera in which the inner detector ring has three-dimensional position resolution and is constructed of a material designed to preferentially Compton scatter the $511 \mathrm{keV}$ annihilation photons. Each annihilation photon may undergo a number of interaction sequences in which the full energy is deposited in the detector system. The two most likely sequences are (1) a single Compton scatter in the solid-state detector followed by absorption in the scintillation detector and (2) absorption in the scintillation detector with no prior interaction in the solidstate detector. More rarely, a $511 \mathrm{keV}$ photon can have a photoelectric interaction in the inner ring. Initial interactions in the inner ring are most desirable because the corresponding end of the annihilation chord can be located precisely. This is especially true for events that Compton scatter since the interaction can be localized within the Compton recoil electron range if the detector pixel size is sufficiently small. Detectors having reasonable sensitivity and depth-of-interaction resolution can be built up using multiple thin layers of planar detectors. 


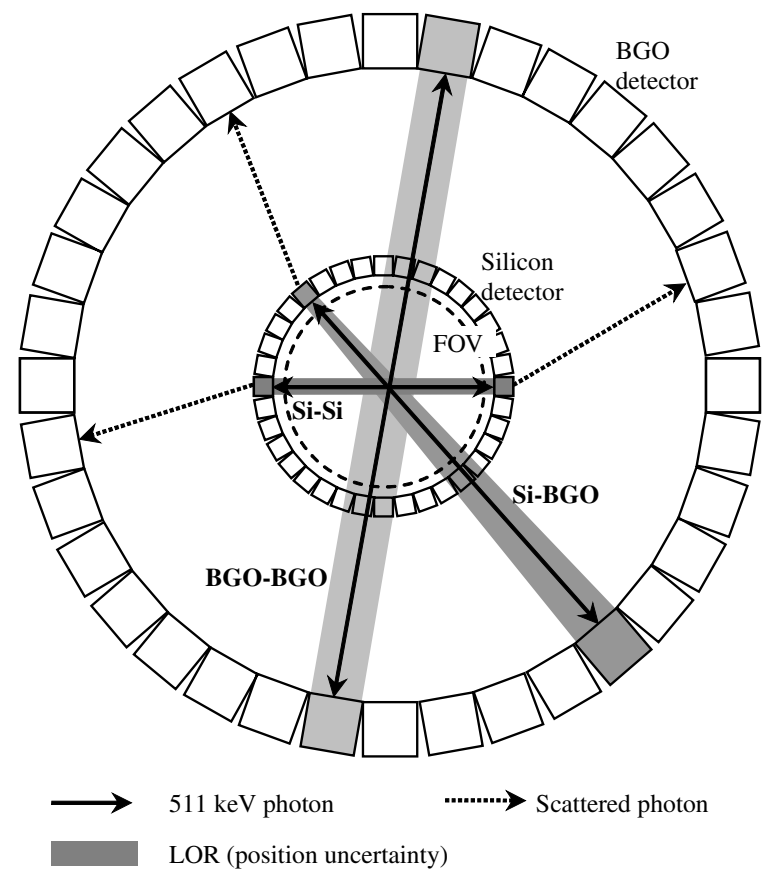

Figure 1. Coincidence events of Compton PET: Si-Si (very high resolution event), Si-BGO (high resolution event) and BGO-BGO (low resolution event).

From the above discussion, interactions in which each of the annihilation photons undergoes a single Compton scatter in the solid-state detector followed by full absorption in the conventional scintillation detectors are most desirable and provide both the highest spatial resolution and the possibility for energy discrimination using the sum of the energies deposited in the inner and outer ring. Since we have been investigating silicon as a material for the inner ring, we term these $\mathrm{Si}-\mathrm{Si}$ events. Of the desirable coincidence events, those where both annihilation photons do not interact in the inner ring and are subsequently fully absorbed in the outer-conventional PET_ring result in the lowest spatial resolution. Again, since we have chosen BGO as the scintillator in our analysis, we term these BGO-BGO events. Finally, there is the hybrid $\mathrm{Si}-\mathrm{BGO}$ event where one annihilation photon interacts first in the inner detector and the other interacts only in the scintillation detector. This process will give intermediate spatial resolution since the energy is deposited over a larger volume in the scintillation crystal, but the other end of the line-of-response (LOR) is accurately determined in the solid-state detector pixel. Figure 1 shows the Compton PET concept using silicon and $\mathrm{BGO}$ as the solid state and scintillation detectors, respectively.

We have presented the Compton PET system and estimated its performance using Monte Carlo simulations (Park et al 2001a, 2002, 2004a). The system for simulations consists of two concentric rings, an inner ring for silicon scatter detector $(4 \mathrm{~cm} \mathrm{ID,} 4 \mathrm{~cm}$ length, and $1.6 \mathrm{~cm}$ thickness composed of 16 layers of $300 \mu \mathrm{m} \times 300 \mu \mathrm{m} \times 1 \mathrm{~mm}$ pads), and an outer ring for conventional BGO PET (17.6 cm ID, $16 \mathrm{~cm}$ length and $2 \mathrm{~cm}$ thickness segmented into $3 \mathrm{~mm} \times 3 \mathrm{~mm} \times 20 \mathrm{~mm}$ crystals). The simulations present a very high resolution of $340 \mu \mathrm{m}$ FWHM with $1.0 \%$ sensitivity at the center of the field-of-view (FOV) from the Si-Si coincidence events and a high resolution of $1.0 \mathrm{~mm} \mathrm{FWHM}$ with $9.0 \%$ sensitivity from the SiBGO events. Conventional PET resolution, $1.66 \mathrm{~mm}$ FWHM with high sensitivity $21 \%$ was 
obtained from the BGO-BGO events. The detection sensitivities of the system as a function of radial point source position for the three event classes were uniformly distributed in the FOV. We also presented that Compton kinematics information in the proposed system provides a method to significantly improve the noise equivalent counts rate (NECR) of the scanner by rejecting random coincidences and photon pairs in which either one or both have been scattered in the object (Park et al 2004b). Since the Compton scattering angle can be estimated from the silicon energy and the position of the scattered photon is recorded by the second detector, one has independent information about the direction of the second annihilation photon such that it must lie within the back-projected conical shell. For this reason, the LORs of only true coincidences lie on the surface of the cone, but those of random and scatter events do not.

The ultimate goal of this work is to develop a very high resolution small animal PET instrument which can achieve sub-millimeter spatial resolution using these concepts. The objective of the immediate investigation is to evaluate the potential for achieving submillimeter spatial resolution at sensitivities comparable to small animal PET scanners of more conventional design. The study reported here was conducted using a laboratory PET instrument made with a pair of position-sensitive silicon detectors.

\section{Materials and methods}

\subsection{Description of prototype system}

In order to demonstrate the sub-millimeter spatial resolution performance with the very high resolution $\mathrm{Si}-\mathrm{Si}$ events, the prototype experimental setup was constructed. Figure 2(a) shows the prototype of a single-slice Compton PET instrument. The system is composed of two silicon pad detectors, four BGO block detectors, a source turntable, tungsten slit collimator and lead shielding. Electronics include a NIM coincidence unit, and silicon and BGO readout systems.

The silicon pad detectors developed for the Compton PET detector are shown at the right of the photograph in figure 2(b). The sensors were processed by SINTEF (Studen et al 2003) and consist of an array of $32 \times 16=512$ pads at a pitch of $1.4 \mathrm{~mm} \times 1.4 \mathrm{~mm}$ with $1 \mathrm{~mm}$ thickness. The detector is rectangular in shape with dimensions of $4.5 \mathrm{~cm} \times 2.2 \mathrm{~cm}$. Four VATAGP3 application-specific integrated circuit (ASIC) chips developed by IDEAS for the low-noise selftriggering readout system were mounted and wire-bonded along one side of the silicon sensor (Studen et al 2004). They are shown at the center of the photograph in figure 2(b). Each readout ASIC provides 128 channels of analog signal shaping and amplification with a multiplexed differential output.

A BGO block detector scavenged from a CTI 931 PET scanner is shown in figure 2(c). The block size is $5.3 \mathrm{~cm} \times 4.9 \mathrm{~cm} \times 3 \mathrm{~cm}$. Four HAMAMATSU PMTs (R2497) were coupled to the BGO block. The detector block consisted of a $4 \times 8$ array of $12.5 \mathrm{~mm} \times 5.25 \mathrm{~mm} \times 30 \mathrm{~mm}$ crystals since it was originally designed for position sensing. This capability was not used in these initial measurements since we only wished to demonstrate the very high resolution performance of the Si-Si events. However, the sum of the four PMT signals can be used to form a timing pulse and for measurement of the photon energy scattered from a silicon detector.

The silicon detectors were located edgewise on opposite sides of a $4 \mathrm{~cm}$ FOV to maximize sensitivity and mounted in black plastic boxes fixed on the platforms for light shielding and mechanical protection (see figures 2(a) and (d)). Geometric alignment was performed by tilting and adjusting platform position while illuminating the edge of the silicon detector through the $1 \mathrm{~mm}$ wide tungsten slit with a horizontal laser beam. Two non-position sensitive BGO detectors flanked each silicon detector for additional timing information and energy 


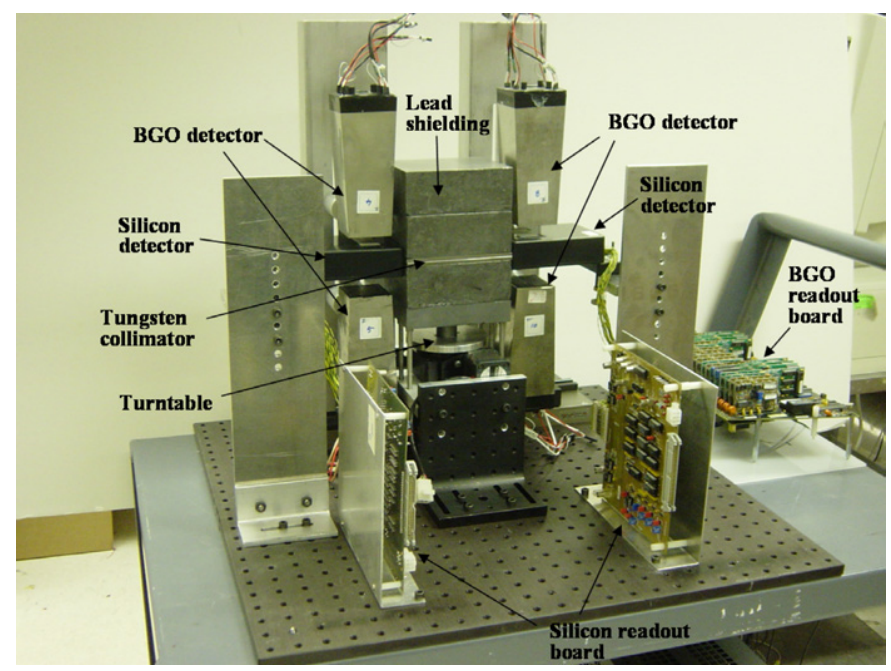

(a)

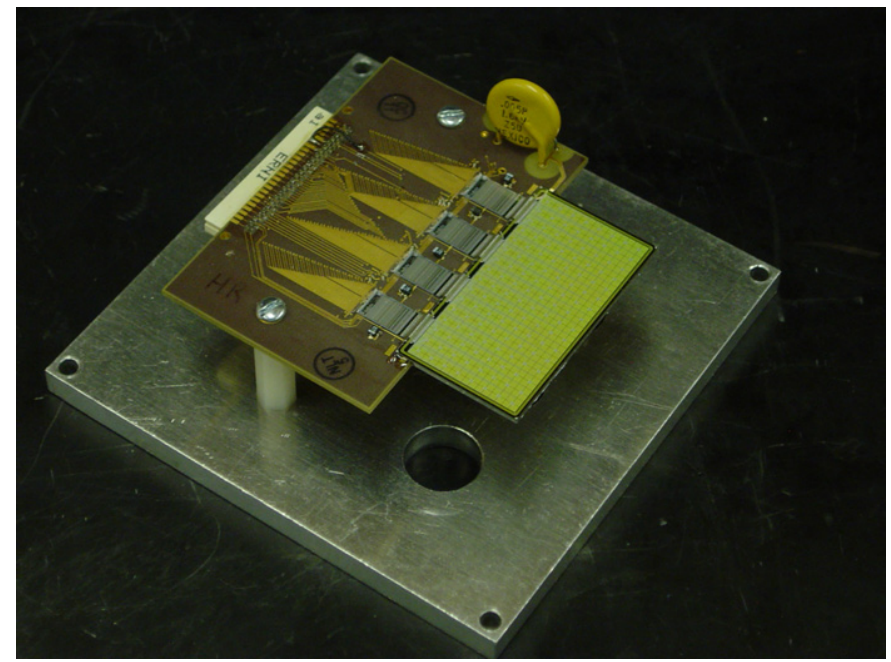

(b)

Figure 2. (a) Prototype Compton PET system consisting of two silicon pad detectors in black plastic boxes, four BGO detectors, source turntable, tungsten slit and lead shielding. (b) Silicon pad sensor ( $32 \times 16$ array of $1.4 \mathrm{~mm}$ square pads and $1 \mathrm{~mm}$ thickness) manufactured by SINTEF and four VATAGP3 128-channel readout chips. (c) BGO crystal $(4 \times 8$ array of $12.5 \mathrm{~mm} \times 5.25 \mathrm{~mm}$ crystals and $3 \mathrm{~cm}$ thickness) coupled with four HAMAMATSU photomultiplier tubes (PMT R2497). (d) Silicon detector alignment using laser beam.

discrimination. To reduce singles rates in the BGO detectors, the source was shielded with a lead block and collimated with two $3 \mathrm{~mm}$ thick tungsten slabs with a $1 \mathrm{~mm}$ gap. Unexpectedly, the source shielding geometry generates large amounts of scatter and the source located out of the slit causes very large numbers of random events. Both scatters and randoms seriously increase the number of singles in the silicon detectors. However, using coincidence timing window and energy discrimination, the contribution of the scatter and random events to coincidence events can be significantly reduced. 


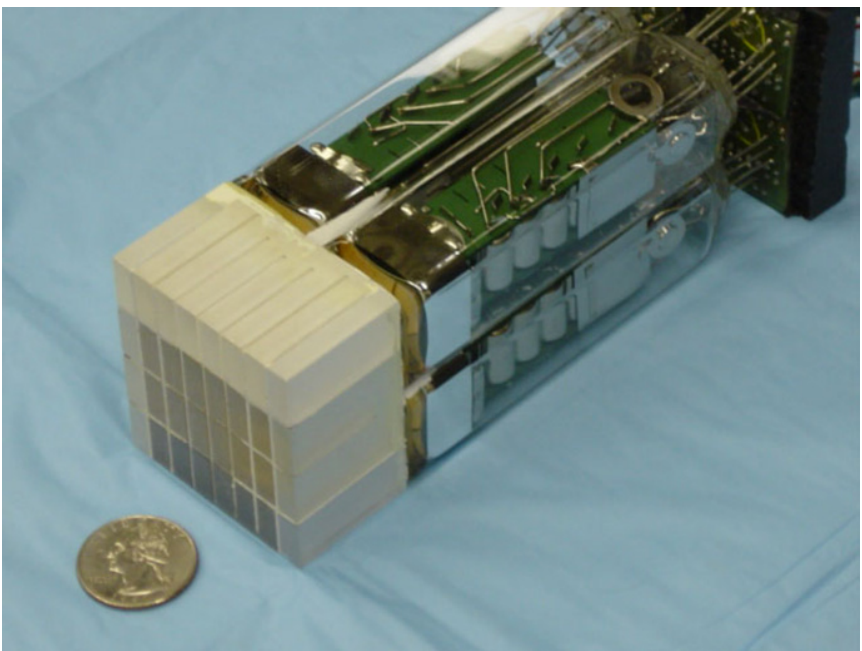

(c)

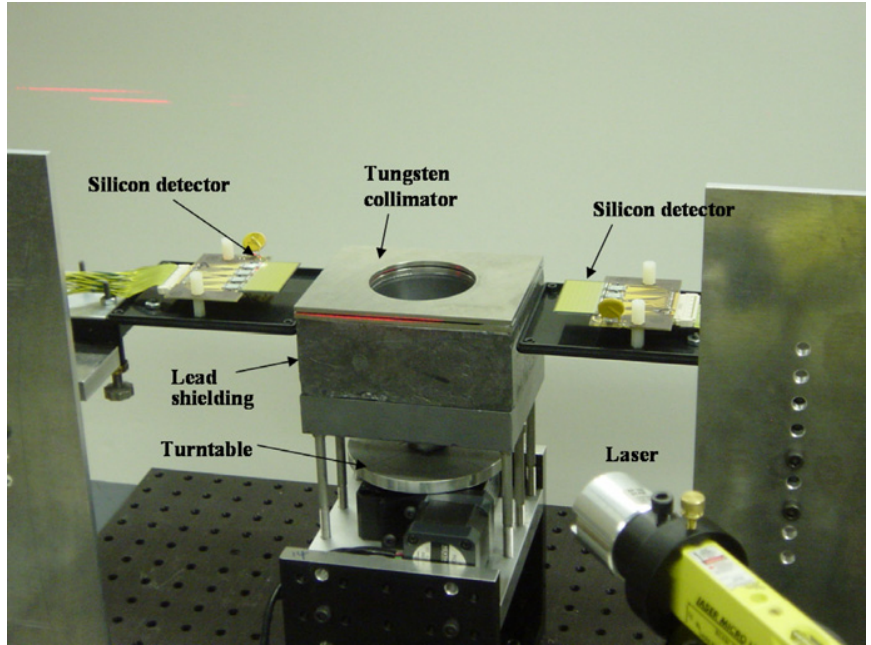

(d)

Figure 2. (Continued.)

Timing pulses from the silicon detector were generated from the trigger ASIC (TA) section of the VATAGP3. The timing pulses from the BGO detector were generated by a constant fraction discriminator (CFD, EG\&G ORTEC 935 quad constant fraction $200 \mathrm{MHz}$ discriminator) that triggers on the summed energy signal from four PMTs. The common silicon trigger from each detector passed through a timing alignment unit consisting of gate and delay generator. The silicon trigger signal was delayed for timing alignment due to the 200 ns delay of the BGO trigger signal in the CFD. The width of the silicon trigger signal was adjusted to define the coincidence timing window. The silicon and BGO triggers were input to a coincidence unit (EG\&G-ESN CO4010, quad 4-fold logic unit). If an event occurred within the coincidence window, a logic pulse was generated. The pulse led to a full coincidence event readout from VME-DAQ ADC and peak sensing ADC. The VME-based readout unit 
processed and 'sparsified' the raw data (energy from each silicon pad and sum energy from BGO detector). The data were buffered and eventually transferred to a PC in list mode.

\subsection{Scanner performance measurement}

2.2.1. Energy resolution. Since the major event in the silicon detectors is Compton scatter and the energy range of detected Compton recoil electrons in the silicon detectors is 0-340 keV, energy resolution was measured with two lower energy sources $59.5 \mathrm{keV}^{241} \mathrm{Am}$ and $140.5 \mathrm{keV}{ }^{99 m} \mathrm{Tc}$, respectively instead of $511 \mathrm{keV}$ annihilation photon sources. $\mathrm{An}^{241} \mathrm{Am}$ point source was placed several centimeters above the silicon sensors, uniformly illuminating all pads. The discriminator threshold was set at about $30 \mathrm{keV}$. Approximately 5 million events were collected for each measurement. In the data post-processing, the mean baseline offset (pedestal) of each silicon pad and common mode noise for each event were subtracted from the ADC values from all 512 pads of the silicon sensors. Since the silicon detector has 512 independent silicon pads, the energy resolution for each pad was determined with Gaussian fit of the photopeak in energy spectrum and the gain for each pad was calculated using the mean of each fit. The energy resolution of the silicon detector was measured with a spectrum summing all gain-corrected energy spectra. After the experiment, the ${ }^{241}$ Am point source was replaced with a small volume of ${ }^{99 \mathrm{~m}} \mathrm{Tc}$ mounted inside a lead collimator. The energy resolution of the silicon detector was measured at the photopeak of ${ }^{99 \mathrm{~m}} \mathrm{Tc}$ using the same procedure for the ${ }^{241}$ Am source.

In order to measure the energy resolution of the BGO detector, a ${ }^{99 \mathrm{~m}} \mathrm{Tc}$ source was used to uniformly illuminate the BGO block. An energy spectrum was acquired from the sum signal of four PMTs. Since each PMT has a different gain, a gain correction must be performed before summing their outputs. The energy resolution was determined with the full width at half maximum of the ${ }^{99 \mathrm{~m}} \mathrm{Tc}$ photopeak using Gaussian fit. Since the major event in the BGO detectors is photoelectric absorption and the energy range of the detected photons scattered from the silicon detector is $171-511 \mathrm{keV}$, it is necessary to measure the energy resolution at higher energy. For a higher energy source, a ${ }^{22} \mathrm{Na}$ point source (511 keV annihilation photon source) was used. Energy resolution was measured at the $511 \mathrm{keV}$ photopeak energy of the ${ }^{22} \mathrm{Na}$ source.

2.2.2. Timing resolution. Timing measurements were conducted between the two silicon detectors and the BGO detectors on opposite sides using annihilation photons from a ${ }^{18} \mathrm{~F}$ point source located at the center of FOV. Silicon TA trigger signals from the VATAGP3 and BGO timing pulses from CFD were fed into a time-to-amplitude converter (TAC, ORTEC 566). Both silicon detectors accepted all events above a TA low level discriminator (LLD) threshold of $30 \mathrm{keV}$. The CFD LLD thresholds were chosen to be as low as possible but sufficiently high to avoid triggering on noise. A multichannel analyzer (ORTEC MAESTRO MCA) was used to display the timing spectrum by digitizing output pulses of the TAC. The width of the coincidence timing window was determined by the timing spectrum and used for the following experiments.

2.2.3. System sensitivity. Geometric alignment is extremely important for detection sensitivity. After we performed geometric alignment with the laser beam as illustrated in figure 2(d), the detection sensitivity for the Si-Si coincidence events was measured with the two silicon detectors using a ${ }^{18} \mathrm{~F}$ point source. A $1.1 \mathrm{~mm}$ ID glass capillary tube was loaded with $480 \mu \mathrm{Ci}$ of ${ }^{18} \mathrm{~F}-\mathrm{FDG}$ and located at the center of the FOV. The height of the source in the capillary tube was $2.5 \mathrm{~cm}$. The portion of source activity in the $1 \mathrm{~mm}$ gap tungsten slit 
was $19.2 \mu \mathrm{Ci}$. The edge-to-edge distance between the two silicon detectors was $17 \mathrm{~cm}$ as determined by the lead shielding and the spacing of the detector from the front face of the plastic box. Detectors were operated only at $100 \mathrm{~V}$ bias for protection while they deplete at $\sim 180 \mathrm{~V}$. The TA LLD setting was $35 \mathrm{keV}$ on both silicon detectors and used for the following experiments. The number of ORed silicon TA trigger signals from the VATAGP3 chips was counted for $10 \mathrm{~s}$ using an ORTEC 994 CCNIM dual counter and timer. To improve statistical precision, measurement was repeated about 50 times. Using the error propagation for multiple independent counts, the mean counts and standard error were calculated. Note that the trigger rate was measured independently from data acquisition. Since data acquisition from all pads requires appreciable processing time, the number of triggers was different depending on the amount of data stored.

2.2.4. Coincidence measurement. Coincidence data were acquired using the experimental setup with a ${ }^{18} \mathrm{~F}$ source. A plastic tube with $4 \mathrm{~mm}$ inner diameter contained $3.8 \mathrm{mCi}$ of ${ }^{18}$ F-FDG and was approximately located at the center of FOV. The height of the source in the tube was $3 \mathrm{~cm}$. The portion of source activity in the $1 \mathrm{~mm}$ tungsten slit was about $127 \mu \mathrm{Ci}$. A $200 \mathrm{~ns}$ coincidence timing window was applied to measure coincidences. For silicon data readout, serial-mode acquisition which acquires signals from all pads for each event was used. After the data post-processing including pedestal, common-noise and gain corrections, interaction positions were determined with the pads having the maximum signal in both silicon detectors and used to draw the LOR. In order to reduce positioning ambiguity, events having multiple signals in channels caused by multiple scatters or obvious multiple events within the coincidence window were rejected. Nevertheless, we note that some of these data can be used for position estimation using Compton kinematics (Kamae and Enomoto 1989, Kroeger et al 2000). The attenuation coefficient of the $511 \mathrm{keV}$ photons in silicon was estimated with the number of coincidence events in each pad from the first and second silicon detectors.

\subsection{Image studies}

2.3.1. Spatial resolution. Image resolution using a very small size source can directly give the intrinsic PET resolution without deconvolving the source size from the image resolution. A ${ }^{18} \mathrm{~F}$-FDG line source in a $25 \mathrm{G}$ stainless steel syringe needle (ID $0.254 \mathrm{~mm}$, wall thickness $0.127 \mathrm{~mm}$ ) was used for the spatial resolution measurement. Total ${ }^{18} \mathrm{~F}$ source activity in the syringe was $500 \mu \mathrm{Ci}$. The effective source activity - the portion of source activity in the $1 \mathrm{~mm}$ gap tungsten slit-was estimated to be approximately $3 \mu \mathrm{Ci}$ using an activity concentration $55 \mu \mathrm{Ci} \mathrm{mm} \mathrm{mm}^{-3}$ since it was difficult to measure the actual source length in the stainless steel needle. The sources were located at the center of the FOV collimated with a tungsten slit for single-slice imaging. The sources were shielded with lead in order to reduce random coincidence events caused by single count rates in the silicon detector from the portion of the source extending beyond the image plane. The coincidence events in the two silicon detectors were acquired using the $200 \mathrm{~ns}$ coincidence timing window. The BGO detectors which give additional timing information and energy discrimination were not used in the first imaging experiment. The source was rotated $180^{\circ}$ with $15^{\circ}$ steps for complete angular sampling. After data post-processing, the pad having an interaction was determined in each silicon detector. The events having multiple hits caused from multiple scatters or multiple events in the coincidence window were rejected to reduce ambiguity in position estimation as noted above. More events in the first rotation step and fewer events in the last steps were rejected since the number of random coincidences and multiple events were reduced as the 
source activity was decaying. The LOR data drawn with hit positions in both silicon detectors were placed in a sinogram. To avoid sampling artifacts in the reconstructed images, the hit position was 'dithered' or randomly selected from a continuous location within each pad of interaction. Image data were acquired into a 128 by 200 distance-angle sinogram. Each bin size was $0.4 \mathrm{~mm}$ by $0.9^{\circ}$. An image was reconstructed using the $2 \mathrm{D}$ filtered back-projection (FBP) with a ramp filter having a cutoff at the Nyquist frequency. Spatial resolution was measured from the point spread function of the $1 \mathrm{D}$ profile of the reconstructed image.

The same experimental settings were used in the following experiments except for rotation step size and data size at each rotation step. These two parameters need to be determined for each experiment based on the source size, maximum distance from the axis-of-rotation and activity.

2.3.2. Comparison with microPET. In order to directly compare the image resolution of the experimental setup with that of a microPET R4, image data were acquired using a ${ }^{18} \mathrm{~F}$ source in the same shape and reconstructed with the same image reconstruction algorithm.

The microPET based on LSO scintillation detectors consisting of $2.1 \mathrm{~mm} \times 2.1 \mathrm{~mm} \times$ $10 \mathrm{~mm}$ LSO crystals was manufactured by Siemens. The microPET R4 is known to have about $1.8 \mathrm{~mm}$ FWHM spatial resolution at the center of the FOV and $4 \%$ sensitivity. The ${ }^{18} \mathrm{~F}-\mathrm{FDG}$ source was filled in two glass capillary tubes. Each capillary tube contained ${ }^{18} \mathrm{~F}$ solution with an activity of about $510 \mu \mathrm{Ci}$. The height of the source in the capillary tube was $3 \mathrm{~cm}$. The effective source activity which is the portion of source activity in the $1 \mathrm{~mm}$ gap tungsten slit was $34 \mu \mathrm{Ci}(17 \mu \mathrm{Ci} \times 2$ tubes $)$. The inner diameter of the capillary tube was $1.1-1.2 \mathrm{~mm}$ and the glass wall thickness was $0.2 \mathrm{~mm}$. The two sources were separated by $0.4 \mathrm{~mm}$, which is the thickness of the glass walls of the tubes. The center-to-center distance between two sources was $1.5 \mathrm{~mm}$. The image data were acquired with the microPET for $5 \mathrm{~min}$. The 350$650 \mathrm{keV}$ energy window and $6 \mathrm{~ns}$ timing window were applied to the system to reject scatter and random events. An image was reconstructed using the FBP with a ramp filter having a cutoff at the Nyquist frequency. In addition, the 3D maximum a posteriori (MAP) algorithm (Qi et al 1998) with 30-iteration was used to obtain the best image (low noise and high resolution image) for image reconstruction. The 3D MAP which is one of the commercial packages available for the microPET has some resolution recovery by modeling annihilation photon pair acollinearity, inter-crystal scatter and penetration which affect detector response.

After the $5 \mathrm{~min}$ acquisition using the microPET, the same source was imaged using the experimental setup of the Compton PET. The acquired data file size was fixed with approximately 4000 coincidence events at each of the 12 rotation steps. In order to compare two different systems, the same image reconstruction method (FBP) was used. Moreover, the maximum likelihood expectation maximization (ML-EM) algorithm (Rockmore and Macovski 1976, Shepp and Vardi 1982, Lange and Carson 1984, Clinthorne et al 2003) without a regularization technique was used to reconstruct the image. The algorithm, which made no specific attempt to accurately model the system response, was stopped at 20 iterations before the noise in the data significantly affected the image. Note that the early stopping of the MLEM iterations can be considered as a form of regularization. Since the ML-EM method was developed to reconstruct 2D images for the experimental setup of the Compton PET system, it cannot be used for the 3D image data from the microPET.

2.3.3. Resolution uniformity. Due to the depth-of-interaction (DOI) uncertainty resulting from the penetration of annihilation photons in a conventional PET detector module, resolution uniformity is one of the important characteristics of PET scanners. In order to examine the resolution uniformity across the FOV of the prototype setup, image resolution was evaluated 
with multiple line source images. The source consists of five line sources located at the center of the FOV and $r=2,5,10$ and $20 \mathrm{~mm}$ from the center. Glass capillary tubes were filled with ${ }^{18} \mathrm{~F}-\mathrm{FDG}$. The height of the source in each capillary tube was approximately $2.5 \mathrm{~cm}$. The height of each source was not carefully measured. However, the effective activity of each source was intended to be identical and was about $21 \mu \mathrm{Ci}$. The source was rotated with $10^{\circ}$ steps.

In order to demonstrate image quality as well as resolution uniformity, the image of four pairs of line sources was reconstructed. The height of the ${ }^{18} \mathrm{~F}$-FDG source in each capillary tube was about $3 \mathrm{~cm}$. The effective activity of each line source was approximately $20 \mu \mathrm{Ci}$. The gap between two sources was only $0.4 \mathrm{~mm}$ from the glass walls. Four pairs of line sources were located approximately at $5 \mathrm{~mm}$ up, $10 \mathrm{~mm}$ left, $15 \mathrm{~mm}$ down and $20 \mathrm{~mm}$ right from the center of the FOV. The source was rotated $360^{\circ}$ in $5^{\circ}$ steps. Since the experimental setup of the Compton PET system consisting of only two silicon detectors cannot fully surround the object, the coincidence detection probability or joint solid angle of two silicon detectors decreases as a function of radial offset position of the line source. Because of this, the image reconstruction algorithm should be corrected for the distribution of the coincidence detection probability in the FOV. The non-uniform system sensitivity across the FOV was corrected with joint solid angle in the ML-EM image reconstruction algorithm for both experiments. Images were reconstructed with 20 iterations.

2.3.4. Phantom images. A hot spot resolution phantom insert (Model ECT/HOT-MMP/I, Data Spectrum Corp., Chapel Hill, NC, USA) designed for evaluation of small animal imaging systems was modified to investigate the performance of image quality (see figure 7(a)). The body of the phantom is a hollow Lucite cylinder with a $4.5 \mathrm{~cm}$ inner diameter containing a hot spot insert. The $4.4 \mathrm{~cm}$ diameter insert has six sectors of $5 \mathrm{~mm}$ long hot rods of different diameters. Since the activity out of image plane causes significant random coincidence events, the insert was modified to reduce the axial extent of the phantom from $3.4 \mathrm{~cm}$ to $5 \mathrm{~mm}$ for the single-slice Compton PET experimental setup. Figure 7(a) shows the modified hot spot insert. The rod diameters are 1.2, 1.6, 2.4, 3.2, 4.0 and $4.8 \mathrm{~mm}$. Center-to-center spacing of the rods is two times their diameter. Since the current prototype system has low sensitivity, we were unable to acquire enough data to reconstruct the entire resolution phantom before the ${ }^{18} \mathrm{~F}$ source decayed away. In order to investigate the imaging performance, the resolution phantom was imaged with ${ }^{18} \mathrm{~F}$ in the innermost hot rods (1.2, 1.6, 2.4, 3.2, 4.0 and $4.8 \mathrm{~mm}$ diameters) and the $1.2 \mathrm{~mm}$ diameter hot rods only, respectively. Approximately $5.0 \mu \mathrm{Ci} \mathrm{mm}{ }^{-3}$ activity was used to fill each of the above hot spot rods. In both experiments, the object was rotated $360^{\circ}$ in $5^{\circ}$ steps. The appropriate sensitivity distribution in the FOV and random events measured using a delayed coincidence window were included in the 30-iteration ML-EM image reconstruction algorithm. Even though the width of the delayed coincidence window was too wide ( $200 \mathrm{~ns}$, the same as the width of the prompt coincidence window), it worked reasonably well to remove the random events.

\section{Results}

\subsection{Scanner performance measurement}

The energy resolution of each silicon detector was measured as $2.5 \%(1.49 \mathrm{keV})$ and $2.4 \%$ (1.45 keV) FWHM at the $59.5 \mathrm{keV}$ emission of ${ }^{241} \mathrm{Am}$. Using a ${ }^{99 \mathrm{~m}} \mathrm{Tc}$ source, corresponding energy resolutions were $0.99 \%(1.39 \mathrm{keV})$ and $0.98 \%(1.38 \mathrm{keV})$ FWHM at $140.5 \mathrm{keV}$. From the pulse-height histograms of the BGO block detectors, nominal energy resolutions of $65 \mathrm{keV}$ FWHM $(46 \%)$ at the $140.5 \mathrm{keV}$ energy of ${ }^{99 \mathrm{~m}} \mathrm{Tc}$ and $116 \mathrm{keV} \mathrm{FWHM} \mathrm{(23 \% )} \mathrm{at} \mathrm{the}$ 


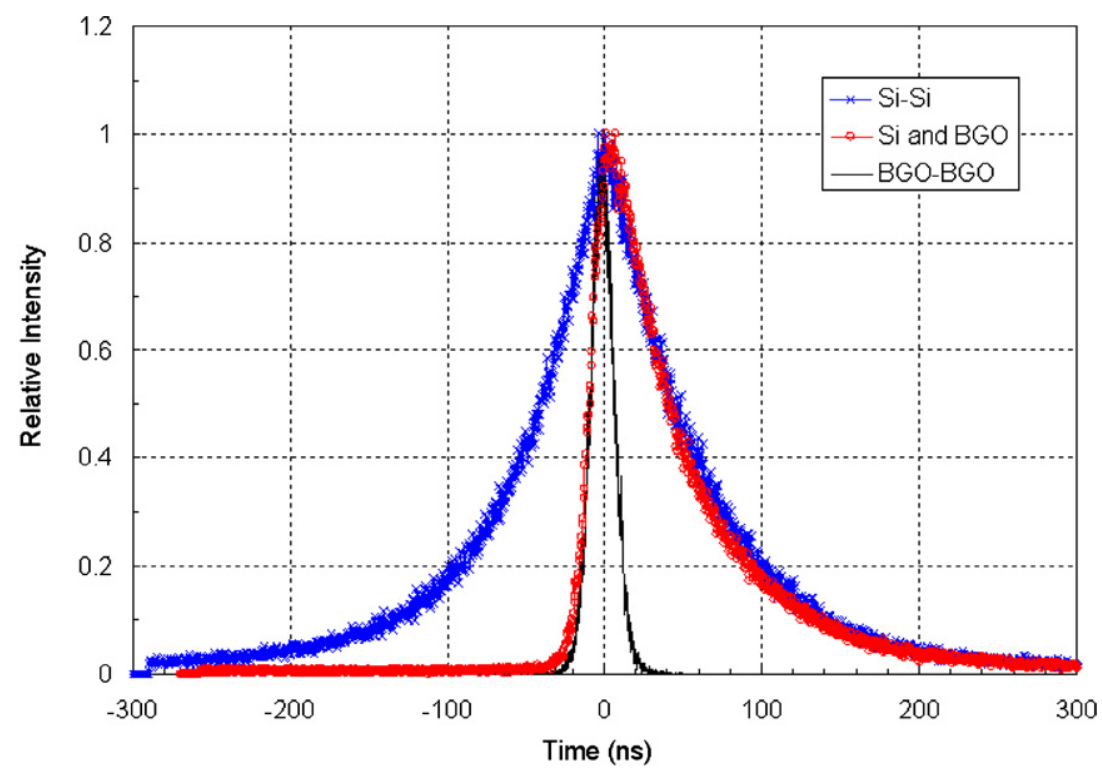

Figure 3. Coincidence timing spectra (timing resolution) from only two silicon detectors ( $82 \mathrm{~ns}$ FWHM), BGO detectors on the opposite sides (19.4 ns FWHM), and a silicon and BGO detectors on one side of the experimental setup using a ${ }^{18} \mathrm{~F}$ source.

$511 \mathrm{keV}$ photopeak energy of the ${ }^{22} \mathrm{Na}$ source were measured. The silicon detectors have excellent energy resolution due to the large number of information carriers (electron-hole pairs) coupled with low electronic noise. However, overall energy resolution is dominated by the BGO detector since the total energy deposited in the system is measured with the sum of silicon and BGO energies for each photon. Although the low energy resolution of BGO restricts the total energy resolution, the energy sum still can be used to reject annihilation photons that have scattered through large angles in the object.

Figure 3 shows the acquired timing spectra using trigger signals of the two silicon detectors and the BGO detectors. The timing spectrum from the only two silicon detectors shows a timing resolution of $82.1 \mathrm{~ns}$ FWHM. The poor timing resolution of the silicon detector is a direct result of the wide energy range of detected Compton recoil electrons (30-340 keV) coupled with the simple leading-edge threshold trigger of present VATAGP3 chips. This is also referred to as 'time-walk'. Due to the time-walk, a wide coincidence timing window (200 ns) was desirable to achieve high detection sensitivity for true annihilation pairs at the cost of high random rate. Using scattered photons from the silicon detectors, a timing measurement made between the BGO detectors on the opposite sides shows a timing resolution $19.4 \mathrm{~ns}$ FWHM. The timing uncertainty ( $\sim 5 \mathrm{~ns}$ FWHM) of a single BGO detector triggering on the $511 \mathrm{keV}$ photons was degraded as a result of the lower signal-to-noise ratio in the BGO detector when triggering on the lower-energy scattered photons. The BGO detector timing pulses from the CFD were fed into 'start' input of the TAC and the silicon trigger signals delayed by $256 \mathrm{~ns}$ were fed into 'stop' input of the TAC. Figure 3 shows an asymmetrical coincidence timing spectrum resulting from different timing uncertainties in the silicon and BGO detectors. The short, sharp tail on the left side of spectrum was from the BGO and the long tail on the right side came from the silicon detector.

From the repeating measurements to estimate coincidence sensitivity using a $200 \mathrm{~ns}$ coincidence timing window, coincidence counting rates were measured at $7.4 \pm 0.8 \mathrm{cps}$. 
Since the well counter which was used to measure the source activity was well calibrated, we assumed that the counting error of the well counter was negligibly small. The detection sensitivity for the $\mathrm{Si}-\mathrm{Si}$ coincidence events, which includes geometric sensitivity (the solid angle subtended by the two silicon detectors) and intrinsic detector sensitivity, was calculated as $1.04 \times 10^{-3} \%\left(\sigma=0.11 \times 10^{-3} \%\right)$.

Figure 4(a) shows a 2D plot of the number of coincidence events in each pad from the first and second silicon detectors. This is also referred to as the '2D hit map'. The 2D hit map shows that the source was located at 3-4 mm below the center of FOV. Since there is no opposing detector element for the off-axis source, there should not be coincidences at the top of both silicon detectors. Nevertheless, a few counts were measured in these channels. We assume that these counts occurred due to random coincidences. The five channels (channels 21, 224, 225 and 384 in the first silicon detector and channel 191 in the second silicon detector), which had no events on the 2D hit map, were masked off before the pedestal and gain calculation for each detector. Figure 4(b) illustrates a LOR plot from 1000 coincidence events. The plot verifies that the events measured in the pads which have zero coincidence detection probability are likely random coincidences as noted above. The 2D hit map in figure 4(a) shows that more events occur at the front of each silicon detector and the number of interactions is exponentially decreasing with silicon detector thickness due to the attenuation. Figure 4(c) illustrates the exponential transmission curve of the annihilation photons in the silicon detectors. The number of transmitted photons was estimated with the number of the events measured in the silicon pads. The top four rows in both arrays and the rows having the channel masked off above were excluded in this attenuation calculation. Using an exponential fit, the attenuation coefficient of the $511 \mathrm{keV}$ photons was measured as $0.1982 \mathrm{~cm}^{-1}$ and $0.1969 \mathrm{~cm}^{-1}$ in the first and second silicon detector, respectively. The measured attenuation coefficients are in good agreement with the value $\left(0.2 \mathrm{~cm}^{-1}\right)$ from the literature (Clinthorne et al 2000).

\subsection{Image studies}

The spatial resolution from the very high resolution $\mathrm{Si}-\mathrm{Si}$ events was measured to be $980 \mu \mathrm{m}$ FWHM from the 1D profile of the $0.254 \mathrm{~mm}$ diameter line source image using Gaussian fit. The image was reconstructed with the FBP. The resolution obtained from the experimental system is slightly different with a theoretical predicted resolution of $700 \mu \mathrm{m}$ FWHM from the triangular response which is half of the $1.4 \mathrm{~mm}$ silicon pad width $(d)$. The maximum resolution loss from annihilation photon acollinearity occurs at the center of the FOV. The contribution to the width of the point spread function is well described by $0.0022 \times$ detector ring diameter $(D)$ with units of millimeters. Since the silicon detectors are separated by $170 \mathrm{~mm}$ in the experimental device, the acollinearity contribution ( $0.374 \mathrm{~mm}$ FWHM Gaussian) is not negligible for this geometry. Moses et al (1997) have proposed a reasonably accurate estimation for the reconstructed image resolution of an arbitrary PET system using FWHM $=1.25 \sqrt{(d / 2)^{2}+(0.0022 D)^{2}+R^{2}+b^{2}}$, where $R$ is the effective source size or positron range and $b$ is a factor due to the crystal decoding process ( 0 if the crystal of interaction is individually coupled to a photo-detector and 2.2 otherwise). The factor of 1.25 is obtained empirically due to reconstruction. Using this equation, the $0.7 \mathrm{~mm}$ FWHM triangular response is convolved with the acollinearity contribution ( $0.374 \mathrm{~mm}$ FWHM) and the effective source size. We assumed that the effective source size including the positron range of ${ }^{18} \mathrm{~F}$ is the source diameter $(0.254 \mathrm{~mm})$ since the source was contained in the dense material (stainless steel needle). The decoding factor is 0 due to the individual silicon pad readout. From the theory, the reconstructed image resolution was estimated as $1.04 \mathrm{~mm}$ FWHM. Therefore, the result from the experiment (980 $\mu \mathrm{m}$ FWHM) 


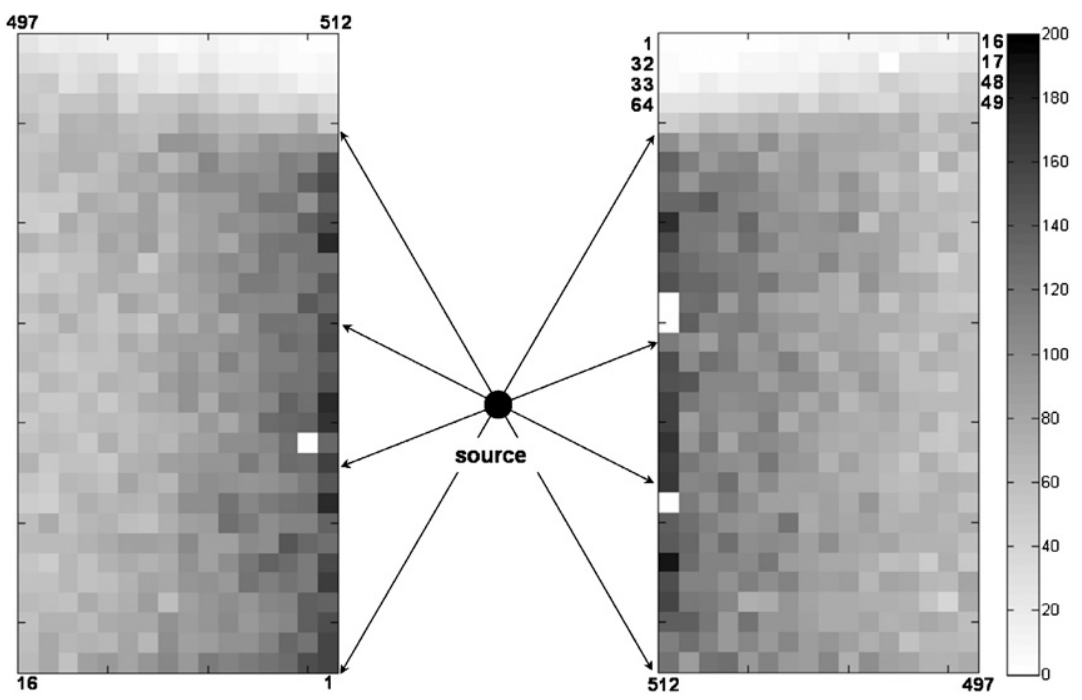

(a)

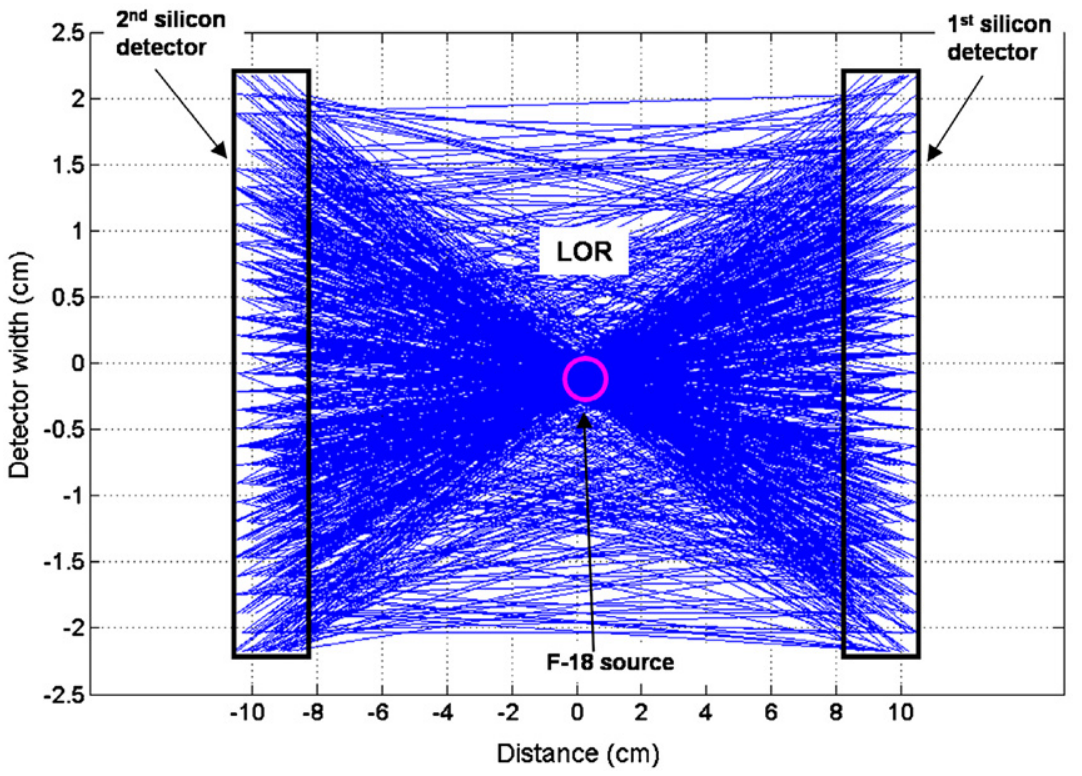

(b)

Figure 4. (a) $2 \mathrm{D}$ plot of the number of coincidence events in each pad or 2D hit map from the first (right) and second (left) silicon detectors. The source is located at a few millimeters below the center of the FOV. The numbers around the silicon detectors are pad or channel numbers in a readout sequence. (b) LOR plot from 1000 coincidence events using the first (right) and second (left) silicon detectors. (c) Exponential transmission curve for $511 \mathrm{keV}$ photons in the silicon detectors. $I_{0}$ is the intensity of photons striking the silicon detector. $I$ is the intensity of the photons that penetrate the silicon thickness without interacting. The attenuation coefficient was estimated using exponential fit.

is in good agreement with the predicted value (1.04 mm FWHM) from the theory. We expect that smaller detector pads coupled with a smaller detector separation will result in significantly higher resolution. 


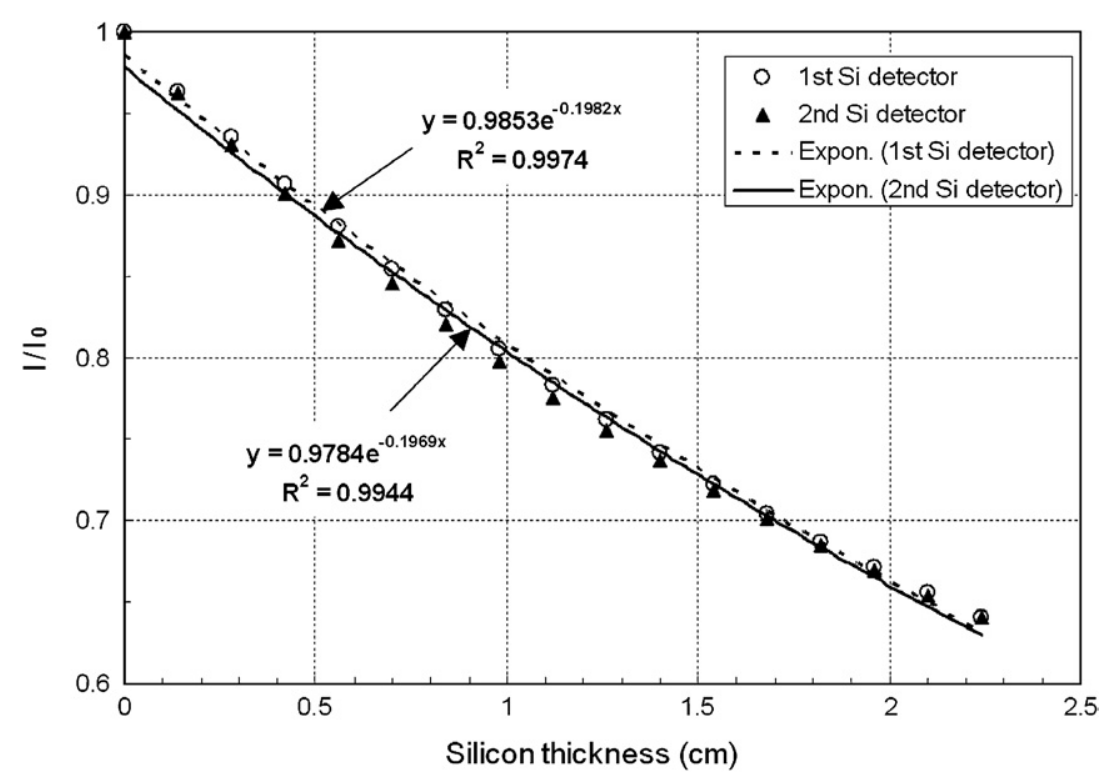

(c)

Figure 4. (Continued.)

Reconstructed images of the two line sources are shown in figure 5. Each image has the same size $5 \mathrm{~cm} \times 5 \mathrm{~cm}$. The image pixel size of the experimental setup is $0.4 \mathrm{~mm}$, while the pixel from the microPET is $0.6 \mathrm{~mm}$ since the original images from the microPET consist of 128 by 128 pixels with $76.8 \mathrm{~mm}$ FOV. The images in figures 5(a)-(c) were obtained from the experimental setup. The two line sources can be resolved at somewhat marginal level in the image reconstructed from the FBP in figure 5(a). However, figures 5(b) and (c) show that the two sources are clearly resolved in the image reconstructed using the ML-EM algorithm. The center-to-center distance of the two line sources on the image from the setup is $1.5 \mathrm{~mm}$ in good agreement with the measured distance.

However, it is impossible to separate the two sources with the images of the microPET in figures 5(d)-(f) because the gap between two line sources is just $0.4 \mathrm{~mm}$ which is approximately four to five times less than the spatial resolutions (1.8 $\mathrm{mm}$ FWHM) of the microPET. In theory, if there were enough recorded events and a sufficiently accurate model of the measurement properties of the tomograph were available, the intrinsic response could be deconvolved and the sources separated. However, such resolution recovery would also entail a generally unacceptable amplification of noise in the image even if the necessary models exist.

Table 1 summarizes radial image resolutions and relative image intensities from the reconstructed five-line source image. Results show that image resolution including $1.1 \mathrm{~mm}$ ID source size is uniformly distributed across the FOV between 1.1 and $1.2 \mathrm{~mm}$ FWHM. Since the DOI was known from the $1.4 \mathrm{~mm}$ pixel location in 16 layers of the silicon detectors, nearly uniform resolution in the FOV was obtained. Note that we used a simple geometric system matrix obtained from forward and back-projection and did not attempt to accurately model the system response at this stage of research. Figure 6 shows the sinogram and reconstructed 2D image of four pairs of the line sources. We found that the actual positions of the sources (the centers of the pairs) in the $(x, y)$ coordinates were $(-1.6,7.4),(-10.9,0.4),(-0.4,-16.8)$ and $(20.3,0)$ with units of millimeters. We did not attempt to calculate spatial resolutions from the 


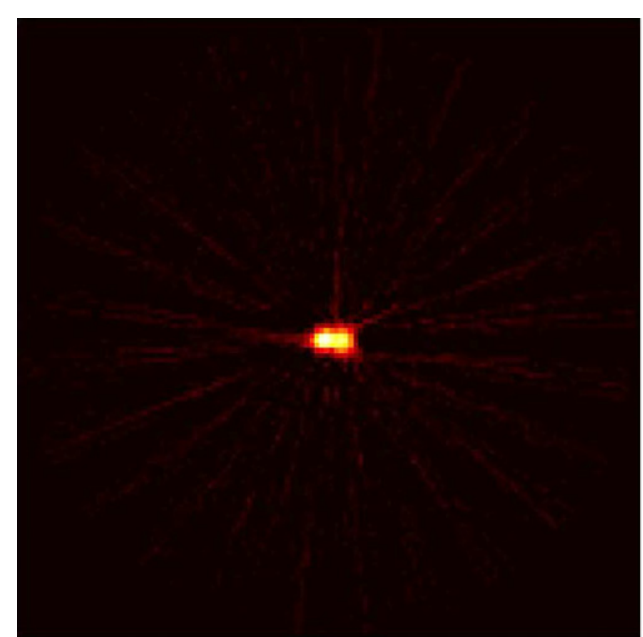

(a)

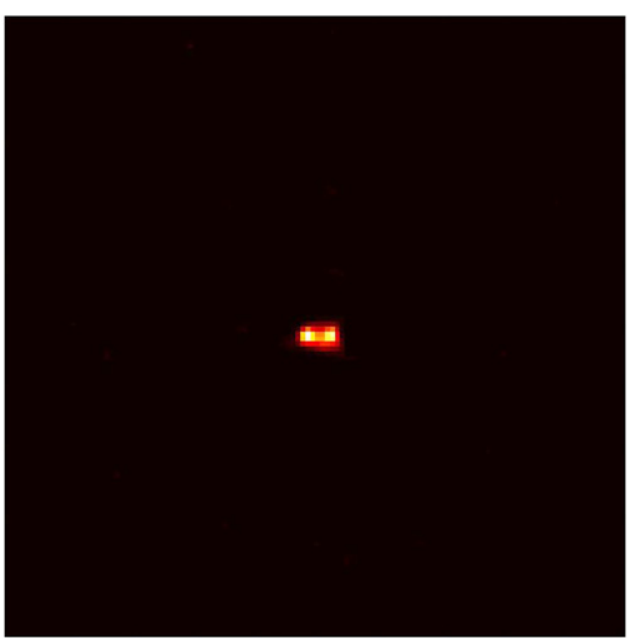

(b)

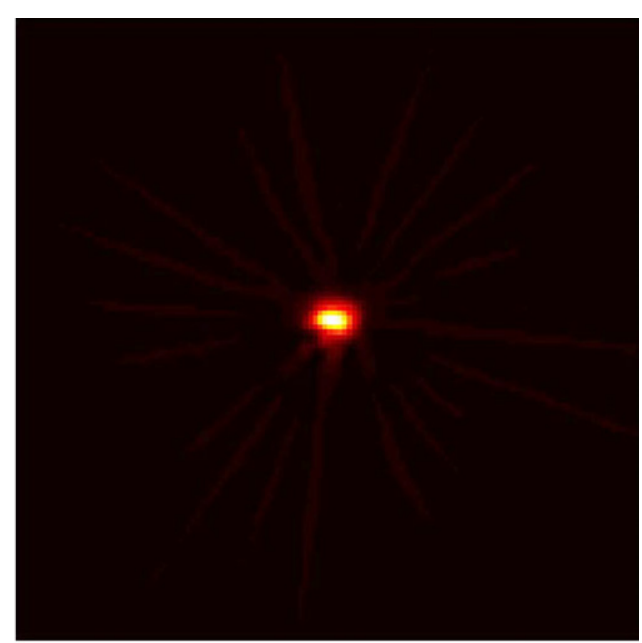

(d)

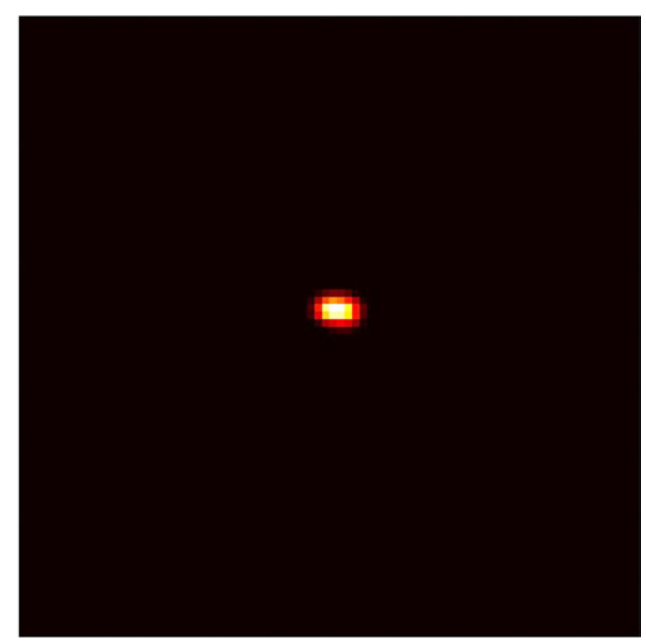

(e)

Figure 5. (a) FBP image, (b) ML-EM image and (c) 1D profiles of the images from the experimental setup. (d) FBP image, (e) MAP image and (f) 1D profiles of the images from the microPET R4. Each image size is $5 \mathrm{~cm} \times 5 \mathrm{~cm}$. Two line sources are ${ }^{18} \mathrm{~F}$-FDG in two $1.1-1.2 \mathrm{~mm}$ ID glass capillary tubes. The gap between two line sources is $0.4 \mathrm{~mm}$ with the walls of the two tubes.

image due to the very small gap $(0.4 \mathrm{~mm})$ between the source pair. However, the prototype Compton PET system clearly resolves each tube of the four-line source pairs at different source positions in the FOV. Moreover, each pair can be resolved even in the sinogram at 0 and $90^{\circ}$ projections. This demonstrates that the high spatial resolution is intrinsic and not due to any resolution recovery 'tricks' of the reconstruction.

Figures 7(b) and (c) show 2D images of ${ }^{18} \mathrm{~F}-\mathrm{FDG}$ in the innermost hot rods and the $1.2 \mathrm{~mm}$ diameter hot rods of the resolution phantom, respectively. Images were reconstructed with the sensitivity and random corrections using the 30-iteration ML-EM. By imaging only part of the resolution phantom to acquire enough data from a small region, the six different size hot rods and smallest hot rods having $1.2 \mathrm{~mm}$ diameters with $1.2 \mathrm{~mm}$ gap are very well resolved 


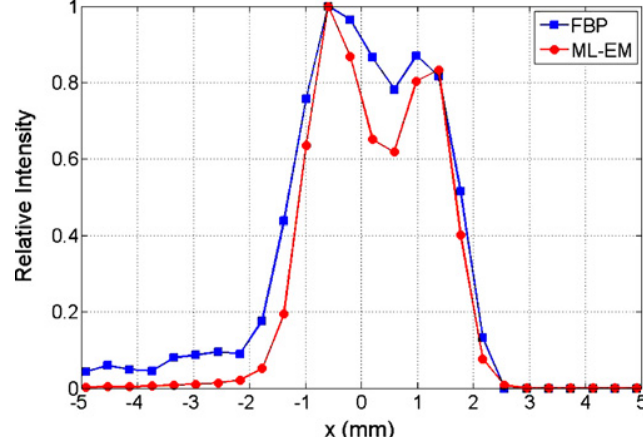

(c)

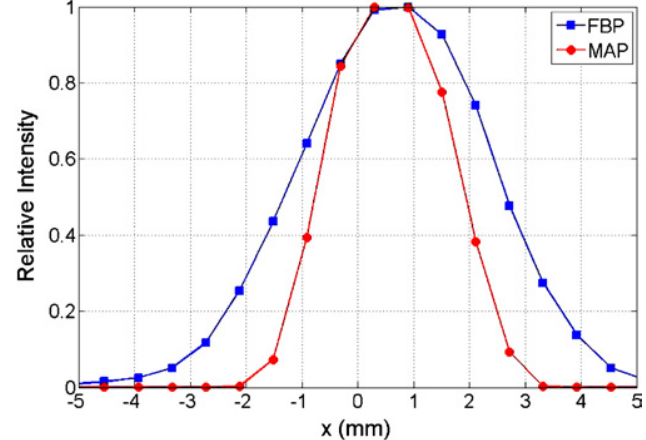

(f)

Figure 5. (Continued.)

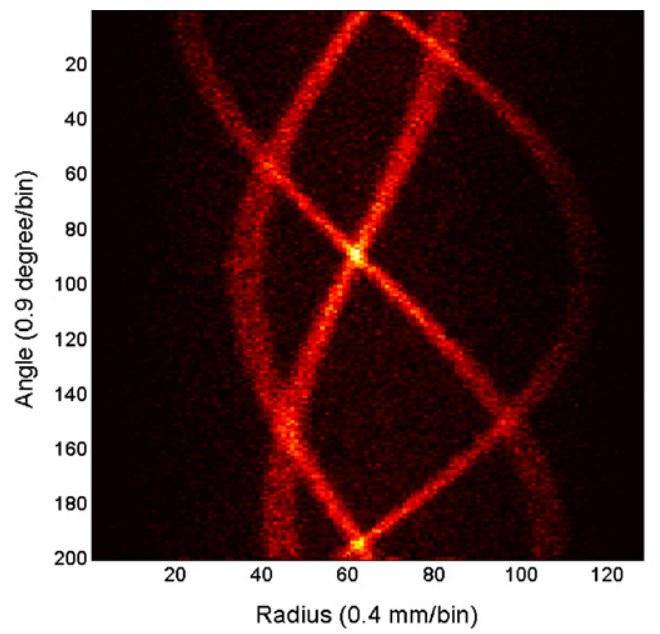

(a)

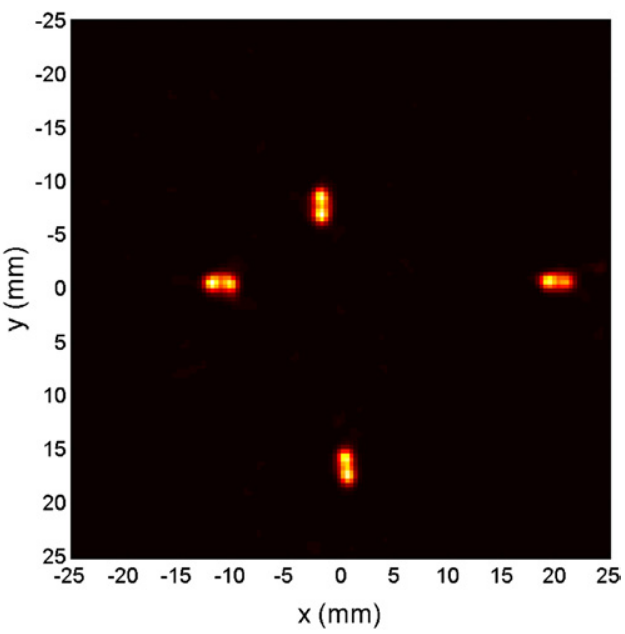

(b)

Figure 6. (a) Image data were acquired into $128 \times 200$ distance-angle sinograms. Each bin size was $0.4 \mathrm{~mm} \times 0.9^{\circ}$. (b) An image reconstructed with 20 -iteration ML-EM using four pairs of ${ }^{18} \mathrm{~F}$ sources in glass capillary tubes (1.1-1.2 mm ID). The gap in each pair is $0.4 \mathrm{~mm}$ with the walls of the two tubes.

Table 1. Relative image intensities and image resolutions of the five line sources.

\begin{tabular}{lrl}
\hline $\begin{array}{l}\text { Radial offset } \\
\text { source position }(\mathrm{mm})\end{array}$ & $\begin{array}{l}\text { Relative intensity } \\
(\%)\end{array}$ & $\begin{array}{l}\text { Radial spatial resolution } \\
(\mathrm{mm} \text { FWHM })\end{array}$ \\
\hline 0 & 89 & 1.11 \\
2 & 100 & 1.03 \\
5 & 95 & 1.10 \\
10 & 80 & 1.18 \\
20 & 97 & 1.08 \\
\hline
\end{tabular}

in the 2D images. One hot rod is not shown in figure 7(c) because it was not fully filled with

${ }^{18} \mathrm{~F}$ due to an air bubble. 


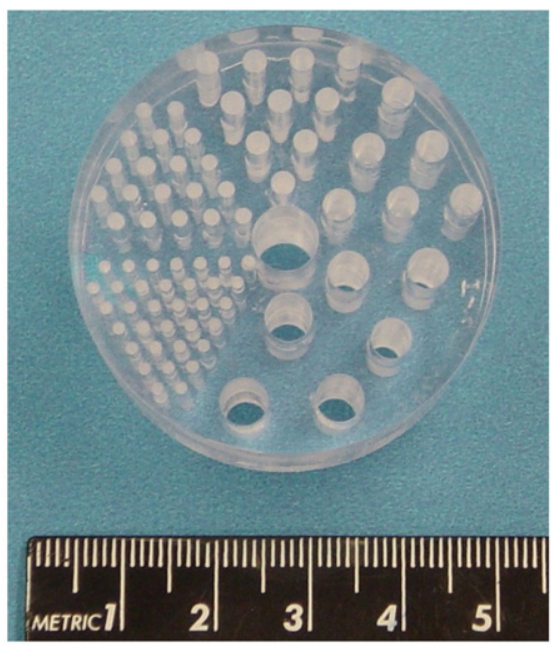

(a)

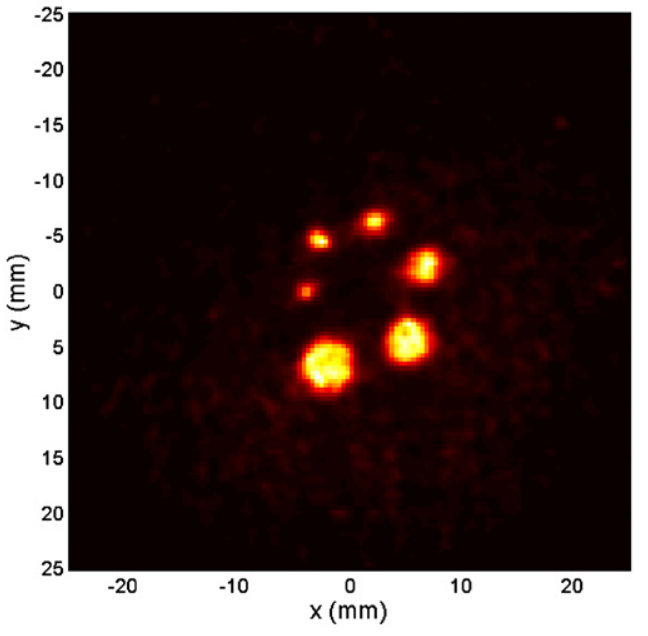

(b)

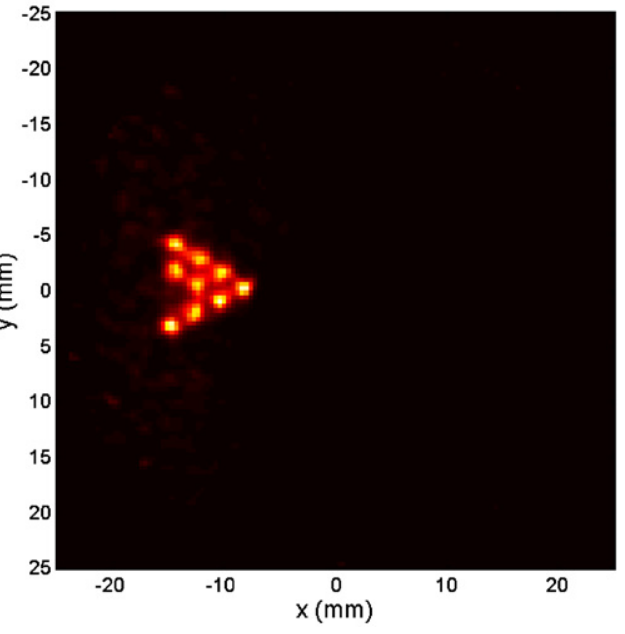

(c)

Figure 7. (a) Resolution phantom (hot spot insert of Micro deluxe phantom). Hot rod diameters are 1.2, 1.6, 2.4, 3.2, 4.0 and $4.8 \mathrm{~mm}$. Center-to-center spacing of rods is two times diameter. (b) An image of a ${ }^{18} \mathrm{~F}$ source in the innermost hot rods $(1.2,1.6,2.4,3.2,4.0$ and $4.8 \mathrm{~mm}$ diameters) of the resolution phantom. (c) An image of a ${ }^{18} \mathrm{~F}$ source in the $1.2 \mathrm{~mm}$ diameter hot rods of the resolution phantom. One hot rod in the left column was not fully filled with ${ }^{18} \mathrm{~F}$ due to an air bubble. Sensitivity distribution and random coincidences were corrected in the 30-iteration ML-EM image reconstruction for both images (b) and (c).

\section{Discussion and conclusions}

It is well known that an ideal PET scanner requires high energy resolution, good coincidence timing resolution, high spatial resolution across the FOV and high sensitivity with low cost in order to achieve the best imaging performance by minimizing random coincidences, detection of scatter from the object, DOI uncertainty, scanning time and radiation dose. From the prototype experimental setup, we have achieved sub-millimeter spatial resolution $(980 \mu \mathrm{m}$ FWHM) at the center of the FOV from the line source image reconstructed with coincidences 
between two silicon scatter detectors ( $\mathrm{Si}-\mathrm{Si}$ events). Excellent resolution uniformity was measured throughout the $4 \mathrm{~cm}$ FOV with minimal DOI uncertainty. However, there are a number of challenges to improve detector and system performance.

The commercial PET scanners have usually less than 10 ns FWHM resolution. A timing window of the commercial scanners is between 2 and $10 \mathrm{~ns}$. Due to the large timing uncertainty of the silicon detectors, however, a wide coincidence timing window (200 ns) is necessary to maintain adequate efficiency for true coincidence events. Besides true events, the wide timing window leads to a high random coincidence rate in the scanner. Present silicon detector units suffer from poor timing resolution. The coincidence timing resolution of the two silicon detectors was 82 ns FWHM due to significant time-walk resulting from the wide range of pulse heights associated with the Compton interactions (30-340 keV) coupled with a simple leading-edge trigger in the current VATAGP3 chip. A new trigger chip being developed with a constant fraction discriminator will significantly improve performance by reducing time-walk. Correcting the timing signal as a function of the recorded pulse height for each event also should greatly improve timing performance.

The current experimental system has a low coincidence detection sensitivity of $1.04 \times$ $10^{-3} \%$ due to the small solid angle subtended by only two opposing silicon detectors. In order to increase sensitivity, silicon detectors should encompass the object being imaged and dense detector packaging is required. Then rotation of neither the detector nor the object is required. In the simple model used in this research, only events that scatter once in silicon and escape were used for imaging since it is difficult to determine which interaction occurred first when there are multiple scatters. However, it is possible to obtain a good estimate of the interaction sequence for many of the multiple scatter events using Compton kinematics when good energy and position information are available. This could significantly improve sensitivity without loss of spatial resolution.

Even though we achieved sub-millimeter spatial resolution from the system, resolution can be improved further since it was limited primarily by the silicon detector pixel size. Nevertheless, small pixel sizes equate with higher costs due primarily to a larger number of electronic channels. In any case, a practical limit to the spatial resolution results from possible migration of the recoil electron between detector pixels and the range of the positron before annihilation in the object. In order to determine the minimum silicon pixel size, further studies are required including additional recoil electron transport simulations in silicon.

Our experimental arrangement was designed to demonstrate the best obtainable spatial resolution from the $\mathrm{Si}-\mathrm{Si}$ events. For this reason, position sensing was not needed in the BGO detectors. In order to demonstrate $\mathrm{Si}-\mathrm{BGO}$ and $\mathrm{BGO}-\mathrm{BGO}$ performance, one must enable position sensing and optimize the BGO geometry as described in figure 1. The complete system could also be implemented by placing densely packed silicon detector modules into a commercial BGO or LSO PET.

Continuing efforts are investigating the above challenges to build a very high resolution small animal PET with high sensitivity. Furthermore, the Compton PET technique can be extended for larger animals or for human imaging applications. The silicon diameters can be scaled up with a loss of sensitivity and some loss of resolution due to acollinearity effects albeit at substantial cost. One might also note that a Compton PET system can also serve as a SPECT instrument for high-energy single photons.

In the previous study, Monte Carlo simulations indicated that a PET instrument based on the Compton PET concept can have spatial resolution which is close to the resolution limit imposed by positron range and annihilation photon acollinearity at sensitivities higher than current small animal PET instruments (Park et al 2001a, 2002, 2004a, 2004b). In this paper, the prototype Compton PET scanner demonstrated sub-millimeter spatial resolution 
performance and excellent resolution uniformity in the FOV. A next setup having sensitivity high enough for cardiac and neurologic studies using mice should allow the achievement of molecular images showing sub-millimeter details.

\section{Acknowledgments}

The authors acknowledge valuable discussions and technical support from the Computer Imaging for Medical Applications (CIMA) collaboration. This work is supported by the United States National Institute of Health under Grant R01 EB000430-34 and R41 RR1513301.

\section{References}

Britz-Cunningham S H et al 2003 Molecular targeting with radionuclides: state of the science J. Nucl. Med. 44 1945-61

Chatziioannou A F et al 1999 Performance evaluation of microPET: a high-resolution lutetium oxyorthosilicate PET scanner for animal imaging J. Nucl. Med. 40 1164-75

Chatziioannou A F et al 2001 Detector development for microPET II: a $1 \mu 1$ resolution PET scanner for small animal imaging Phys. Med. Biol. 46 2899-910

Cherry S R et al 1997 MicroPET: a high resolution PET scanner for imaging small animals IEEE Trans. Nucl. Sci. 44 1161-6

Clinthorne N H et al 2000 Very high resolution animal PET J. Nucl. Med. 41120

Clinthorne N H et al 2003 Multi-resolution image reconstruction for a high-resolution small animal PET device IEEE Nucl. Sci. Symp. Med. Imaging Conf. 3 1197-2001

Correia J A et al 1999 Development of a small animal PET imaging device with resolution approaching $1 \mathrm{~mm} I E E E$ Trans. Nucl. Sci. 46 631-5

Del Guerra A et al 1998 YAP-PET: first results of a small animal positron emission tomograph based on YAP:Ce finger crystals IEEE Trans. Nucl. Sci. 45 3105-8

Huber J S and Moses W W 1999 Conceptual design of a high-sensitivity small animal PET camera with 4 pi coverage IEEE Trans. Nucl. Sci. 46 489-502

Janecek M et al 2004 High resolution insert for clinical whole body PET scanner: design and optimization IEEE Nucl. Sci. Symp. Med. Imaging Conf. 6 3846-52

Kamae T and Enomoto R 1989 Gamma ray measurement utilizing multiple Compton scattering US Patent 4857737

Kroeger R A et al 2000 Three-compton telescope: theory, simulations, and performance IEEE Trans. Nucl. Sci. 49 1887-92

Lange K and Carson R 1984 EM reconstruction algorithms for emission and transmission tomography J. Comput.Assist. Tomogr. 8 306-16

Moses W W et al 1997 Design of a high resolution, high sensitivity PET camera for human brain and small animals IEEE Trans. Nucl. Sci. 44 1487-91

Nichol C and Kim E E 2001 Molecular imaging and gene therapy J. Nucl. Med. 42 1368-74

Park S et al 2001a Design of a very high resolution animal PET J. Nucl. Med. 4255

Park S et al 2001b Experimental setup for very high resolution animal PET based on solid state detector IEEE Nucl. Sci. Symp. Med. Imaging Conf. 2 1120-3

Park S et al 2002 Effect of recoil electron range on sensitivity and on spatial resolution of very high resolution animal PET IEEE Nucl. Sci. Symp. Med. Imaging Conf. 3 1756-9

Park S et al 2004a Effect of positron range and annihilation photon acolinearity on spatial resolution of Compton PET J. Nucl. Med. 45166

Park S et al 2004b Improvement of noise equivalent count rate using Compton kinematics in a Compton PET IEEE Nucl. Sci. Symp. Med. Imaging Conf. 6 3911-5

Park S et al 2005 First experimental results from the very high resolution PET concept J. Nucl. Med. 4651

Qi J et al 1998 High-resolution 3D Bayesian image reconstruction using the microPET small-animal scanner Phys. Med. Biol. 43 1001-13

Rockmore A J and Macovski A 1976 A maximum likelihood approach to emission image reconstruction from projections IEEE Trans. Nucl. Sci. 23 1428-32

Seidel J et al 2003 Resolution uniformity and sensitivity of the NIH ATLAS small animal PET scanner: comparison to simulated LSO scanners without depth-of-interaction capability IEEE Trans. Nucl. Sci. 50 1347-50 
Shepp L A and Vardi Y 1982 Maximum likelihood reconstruction for emission tomography IEEE Trans. Med.Imaging $1113-22$

Studen A et al 2003 Development of silicon pad detectors and readout electronics for a Compton PET Nucl. Instrum. Methods A 501 273-9

Studen A et al 2004 First coincidence in pre-clinical Compton camera prototype for medical imaging Nucl. Instrum Methods A 531 258-64

Tai Y et al 2001 Performance evaluation of the MicroPET R4: a PET system dedicated to animal imaging Phys. Med. Biol. 46 1845-62

Tai Y et al 2003a Design study of a detector insert for high resolution clinical PET imaging IEEE Nucl. Sci. Symp. Med. Imaging. Conf. 3 1714-7

Tai Y et al 2003b MicroPET II: design, development and initial performance of an improved microPET scanner for small animal imaging Phys. Med. Biol. 48 1519-37

Tai Y et al 2005 Performance evaluation of the microPET Focus: a third-generation microPET scanner dedicated to animal imaging $J$. Nucl. Med. 46 455-63

Thanos P K et al 2002 In vivo comparative imaging of dopamine D2 knockout and wild-type mice with ${ }^{11} \mathrm{C}$-Raclopride and microPET J. Nucl. Med. 43 1570-77

Weber S et al 2000 Recent results of the TierPET scanner IEEE Trans. Nucl. Sci. 47 1665-9 